\title{
Análisis de trayectorias de estudiantes y egresadas de nivel posgrado en México: retos y oportunidades en el marco de la emergencia sanitaria por COVID-19
}

\author{
Analysis of the trajectories of students and graduates of postgraduate level in \\ Mexico: challenges and opportunities in the framework of the health emergency \\ due to COVID-19
}

\author{
Fernando Vázquez Torres ${ }^{1}$, Nathalie Vázquez Monter $^{2}$, José Orozco-Santiago ${ }^{3}$ \\ fvazquezt@ipn.mx, nathalie.v.monter@gmail.com, jorozcos@ipn.mx \\ ${ }^{1,3}$ Intituto Politécnico Nacional \\ UPIICSA-SEPI \\ Ciudad de México, México \\ ${ }^{2}$ Investigación y Proyectos Educativos \\ Hipatia México \\ Tequisquiapan, México
}

\begin{abstract}
Resumen-. En las últimas décadas, los programas de posgrado como parte del sistema de educación superior de México, han tenido un crecimiento expansivo, al igual que en otros países de Latinoamérica. Sin embargo, el crecimiento en la oferta y demanda de estos programas no necesariamente garantiza la pertinencia y calidad de éstos. En este sentido, los recursos disponibles para garantizar la movilidad estudiantil, la relación entre la oferta y la demanda de los diferentes programas, la vinculación con los sectores productivos y de servicios, las oportunidades en el ámbito laboral, etc. serán factores que definirán las trayectorias de estudiantes y egresados. A este escenario, se suman las implicaciones de la emergencia sanitaria internacional por COVID19, que afectaron a las instituciones educativas de todo el mundo. En este proyecto se desarrolla una metodología de investigación de campo a través de encuestas dirigidas a estudiantes y egresadas de nivel posgrado en México, integrando métodos cuantitativos y cualitativos para determinar ¿cómo incide la pandemia por COVID-19 y la postpandemia en la formación, movilidad académica internacional e inserción laboral de dichos egresados? y ¿cuáles son los retos y las oportunidades de los nuevos investigadores ante el escenario actual?
\end{abstract}

Palabras clave: pandemia, COVID-19, trayectorias estudiantes, posgrado, retos y oportunidades.

Abstract- In recent decades, postgraduate programmes as part of Mexico's higher education system have experienced expansive growth, as in other Latin American countries. However, the growth in supply and demand of these programmes does not necessarily guarantee their relevance and quality. In this sense, the resources available to guarantee student mobility, the relationship between supply and demand of the different programmes, the links with the productive and service sectors, opportunities in the labour market, etc. will be factors that will define the trajectories of students and graduates. Added to this scenario are the implications of the international health emergency caused by COVID-19, which affected educational institutions all over the world. This project develops a field research methodology through surveys directed at postgraduate students and graduates in Mexico, integrating quantitative and qualitative methods to determine how the COVID-19 pandemic and the post-pandemic affect the training, international academic mobility, and labour market insertion of these graduates, and what are the challenges and opportunities for new researchers in the current scenario?

Keywords: pandemic, COVID-19, student and graduate trajectories, challenges and opportunities

\section{INTRODUCCI ÓN}

En diciembre de 2019 se originó en China una enfermedad viral SARS-CoV-2 (COVID-19) que se convirtió en unos meses en pandemia, con efectos devastadores para todos los integrantes de la sociedad (Yi, Lagniton, Ye, Li, \& Xu, 2020). En el interconectado mundo moderno, la incertidumbre y sorpresa por lo rápido que se ha alterado la vida de prácticamente todos los habitantes del planeta ha creado una situación inédita para todas las organizaciones, incluyendo las instituciones de educación superior, quienes se han visto obligadas a suspender las actividades académicas cara a cara, en una transición abrupta y obligada a la educación a distancia mediada por la tecnología (Sanz, Sáinz González \& Capilla, 2020).

La suspensión obligatoria de las clases en todos los niveles educativos, con el confinamiento en casa de estudiantes y profesores, ha creado una red de efectos múltiples en todos los actores del proceso educativo. En el caso de las universidades, éstas han tenido que tomar una serie de medidas para acatar las indicaciones gubernamentales, al tiempo que intentan proporcionar a profesores y estudiantes la infraestructura informática y tecnológica que les permita continuar con sus actividades académicas a distancia.

Los docentes dejaron el salón de clase tradicional, al que han estado acostumbrados por décadas, para convertirse de manera obligada en usuarios de las herramientas tecnológicas que existen para interactuar a distancia entre ellos mismos y sus estudiantes, al tiempo que tienen que atender las presiones personales del confinamiento y sus implicaciones económicas, de salud y afectivas. 
Por su parte, los estudiantes, que se encuentran súbitamente en su casa, comparten, si es que los hay, los dispositivos digitales y la red de internet que usa toda la familia, y tienen la necesidad de continuar sus actividades de aprendizaje de las diversas asignaturas inscritas, a través de tareas, conferencias virtuales y una serie de deberes que + superponen. Además, tienen que lidiar con los posibles efectos de la pandemia en la salud, las emociones, actividades físicas y las propias de la juventud (The Chronicle of Higher Education, 2020).

Esta combinación simultánea de entornos complejos ha creado una serie de acciones y emociones como nunca habíamos visto, que ha tomado prácticamente por sorpresa a las universidades, la comunidad de profesores, los estudiantes y la sociedad en general. A esto se le agrega la profunda incertidumbre, consecuencia de no saber cómo avanzará la pandemia.

Esta situación, obligada para transitar de la educación universitaria presencial y escolarizada a modalidades no presenciales, mediadas por las tecnologías del aprendizaje y el conocimiento, puso de manifiesto las necesidades institucionales de infraestructura, sobre todo para repensar cómo se quiere que sea la transformación e implementación de la enseñanza, y la nueva cultura y prácticas docentes.

\section{CONTEXTO}

En las últimas décadas, los programas de posgrado como parte del sistema de educación superior de México han tenido un crecimiento expansivo, al igual que en otros países de Latinoamérica (Bonilla, 2015). Sin embargo, el crecimiento en la oferta y demanda de estos programas no necesariamente garantiza la pertinencia y calidad de estos. En este sentido, aspectos como: la agenda en las líneas de investigación, los recursos disponibles para garantizar la movilidad estudiantil, la relación entre la oferta y la demanda de los diferentes programas, la vinculación con los sectores productivos y de servicios, las oportunidades en el ámbito laboral, los medios para el financiamiento, etc. serán factores que definirán las trayectorias de estudiantes y egresados. A este escenario, se suman las implicaciones de la emergencia sanitaria internacional por COVID-19, que durante el año 2020 cimbró la estructura organizativa de las instituciones educativas en todos los niveles, tanto en México como en el resto del mundo.

Este proyecto, pretende desarrollar un estudio que integra métodos cuantitativos y cualitativos para el análisis de las trayectorias de estudiantes y egresados de programas de posgrados en México. De esta manera, el estudio pretende dar respuesta a las siguientes preguntas iniciales de investigación: ¿Cómo incide la pandemia por COVID-19 y la postpandemia en la formación, movilidad académica internacional e inserción laboral de dichos egresados? ¿Cuáles son los retos y las oportunidades de los nuevos investigadores ante el escenario actual?

\section{A. Justificación}

El interés que se tuvo para realizar este trabajo de investigación surge de la necesidad de obtener la mayor información posible sobre los efectos de la pandemia de COVID-19 para poder reformar y reestructurar los planes y programas de estudio de nivel posgrado y replantear las trayectorias de los egresados tanto de maestría como de doctorado.

\section{B. Objetivos}

El objetivo general es identificar los retos y las oportunidades de la Educación Superior en México, específicamente a nivel de posgrado, ante la emergencia sanitaria por COVID-19, a partir de la interpretación y reconocimiento de las perspectivas de estudiantes y egresados de dichos programas de formación.

El objetivo específico es identificar los principales factores que intervienen en la construcción de trayectorias de estudiantes y egresados de posgrado, considerando un mapeo de dichas trayectorias y a través de un análisis cuantitativo enfocado en definir la influencia de las desigualdades económicas y sociales de los participantes en México. A su vez, este análisis deberá definir cuáles son las inserciones en el ámbito gubernamental, educativo y social.

\section{Público objetivo}

Los resultados obtenidos de este trabajo de investigación están enfocados a los administradores educativos, profesores y autoridades del sector educativo que tendrán que considerar un conjunto de políticas educativas diseñadas para apoyar a los sistemas educativos a afrontar los impactos inmediatos provocados por la pandemia de COVID-19, así como plantear oportunidades en el marco de la formación de estudiantes de posgrado.

\section{DESCRIPCIÓN}

Diseño del estudio. El proyecto constó de las siguientes fases: (1) Diseño de la encuesta que contempla el diseño del instrumento de medición y el diseño de muestreo para el levantamiento de la información estadística; (2) Levantamiento de la encuesta propiamente o trabajo de campo; (3) Concretar el estudio por parte de los investigadores, lo que incluye el análisis de los datos mediante la encuesta aplicada a un grupo de egresadas del posgrado en México, así como la elaboración e interpretación de indicadores y todos los elementos necesarios para elaborar el diagnóstico y las recomendaciones para proponer políticas públicas en el posgrado nacional y dar respuesta a nuestras preguntas de investigación.

El proyecto permite disponer de información integral y confiable de los posgrados que se imparten en el país. El Diagnóstico del posgrado nacional beneficiará a los siguientes actores:

- En el sector gobierno, al Consejo Nacional de Ciencia y Tecnología (CONACYT) y al Ministro de Educación en México (SEP), quienes dispondrán de información de la situación actual del posgrado, lo cual les permitirá tomar decisiones de política pública, a partir del conocimiento preciso de las áreas prioritarias a atender.

- En el sector educativo y científico, las Instituciones de Educación Superior y Centros de Investigación; quienes tendrán información actualizada de las fortalezas y debilidades del posgrado nacional, con lo cual podrán rediseñar e implementar acciones de fortalecimiento de la calidad de sus posgrados.

- Para el sector social el posgrado nacional es un tema estratégico para el desarrollo del país que necesita tener un seguimiento puntual y contar con una base de datos actualizada en todo momento para consulta de la sociedad en general. La sociedad contará con programas de calidad 
que generan investigación socialmente relevante y profesionistas que responden a las condiciones del mercado en donde se van a desempeñar.

Metodología. El estudio es de tipo descriptivo, mediante uso de encuesta y entrevistas semi-abiertas. Los informantes son las estudiantes y egresadas de los programas de posgrado seleccionadas en la muestra. Esta primera fase es un primer contacto con la realidad que nos interesa conocer y que permitirá en un futuro llevar a cabo estudios longitudinales que sirvan para dar seguimiento a las trayectorias, estrategias y políticas que se implanten por parte de las instancias de las Instituciones de Educación Superior.

Características de la encuesta:

- Transversal: Se lleva a cabo solo una medición en el tiempo.

- Prospectiva: Se genera información a partir del levantamiento de utilidad para contestar los objetivos del proyecto.

- Descriptiva: No se modifican o controlan variables de interés, pues está centrado en recolectar datos que describen la situación actual.

- Período: abril 2021 - junio 2021.

\section{A. Revisión de la literatura}

Se ha ponderado que el impacto económico del Covid-19 en el mundo puede ser irreversible y catastrófico. Según la Comisión Económica para América Latina y el Caribe (CEPAL, 2020a,b), en su informe especial, las medidas de autoaislamiento, cuarentena y distanciamiento social han provocado efectos directos en la oferta y la demanda: suspensión de actividades productivas y mayor desempleo. Así también, recesión mundial en educación, comercio, turismo, transporte, manufactura y recursos naturales. Esta pandemia, según los expertos en economía, agrava la crisis mundial que se estaba padeciendo desde 2008. Hasta este punto el panorama económico está más que estimado $\mathrm{y}$, conforme a dichas proyecciones, se aplicarán las medidas y estrategias correspondientes para solucionar el problema. Sin embargo, en materia educativa, esto no es así. En educación pareciera no haber una estrategia clara en cuanto a cómo abordar las afectaciones en este ámbito.

Buscamos mostrar el sentir y las voces de los actores de la educación superior desde dentro del proceso formativo, contrastándolos con las disposiciones y sugerencias de organismos internacionales y acuerdos nacionales. Para ello, en un primer momento se señala el impacto económico que se ha desencadenado a causa de la pandemia Covid-19; en segundo término, las consecuencias en educación, esto es, que el contexto sanitario tenga una mayor repercusión en la economía, mientras que en educación sólo se muestra lo evidente con respecto a las afectaciones. Lo importante es evidenciar el sentir, los obstáculos, las competencias desarrolladas y los retos reales que ha producido el ajuste de las clases presenciales a virtuales desde la voz de los estudiantes, docentes y administrativos, con el fin de ser referente para propuestas, programas o planes en educación superior en contextos de pandemia. La metodología utilizada debe ser el análisis de trayectorias de estudiantes de posgrado con diseño mixto, para contrastar entre lo real y lo expresado sobre educación superior por organismos internacionales.

\section{B. Diseño, pilotaje y aplicación de herramientas}

Diseño de instrumento de medición. El cuestionario es un instrumento que se utiliza de un modo preferente, en el desarrollo de investigaciones, en particular en las encuestas. Su construcción, aplicación y tabulación poseen un alto grado de organización y objetividad. El instrumento de medición en este proyecto se construyó en función de los objetivos definidos en el mismo y, en general, fue de tipo restringido o cerrado. En el cuestionario se incluyeron tres secciones de control referentes a: 1) Datos de control Interno del proyecto; 2) Datos de la Institución, y 3) Datos de la egresada de un programa de posgrado.

Finalmente, se obtuvo un cuestionario que contiene 26 variables, al cual se le dio un formato de impresión tipo cuadernillo, de 2 páginas.

\section{Análisis cuantitativo de los datos}

El objetivo de este análisis fue identificar los principales factores que intervienen en la construcción de las trayectorias de los estudiantes y egresados de posgrado, considerando un mapeo de dichas trayectorias y a través de un análisis cuantitativo determinar el contexto en el que los participantes están inmersos.

Este estudio de investigación comprendió un estudio de caso inicial con una muestra de 16 participantes mediante una encuesta y que en las próximas semanas se espera extender a una mayor población mediante invitación por correo institucional, redes sociales, congresos nacionales e internacionales.

Los resultados obtenidos fueron analizados de forma independiente por los investigadores y se negociaron las conclusiones entre ellos para validación.

\section{Análisis cualitativo}

El análisis cualitativo de los datos recabados a través de dos preguntas abiertas en la encuesta (Creswell y Creswell, 2018), estuvo enfocado en determinar los obstáculos enfrentados en los últimos cinco años y aquellos directamente vinculados a la emergencia sanitaria en los últimos dos. Los datos se organizaron y analizaron con la ayuda de un software Atlas.ti.

En ese sentido, el primer paso consistió en codificar las respuestas de las participantes, de acuerdo con criterios establecidos por dos investigadores de forma separada. Posteriormente se contrastaron los criterios para la codificación y se definieron las categorías para los diferentes tipos de obstáculos encontrados durante los últimos cinco años de sus trayectorias y en particular los últimos dos ante la emergencia por Covid-19. Finalmente, se cuantificaron los resultados.

\section{Resultados}

Los resultados se dividen en dos partes: la primera parte, que es un sondeo cuantitativo que permite la representación gráfica de las trayectorias, y la segunda parte, que es resultado de un pilotaje de análisis cualitativo que nos permita conocer con más detalles los obstáculos que los participantes enfrentaron en los 
últimos cinco años y aquellos que fueron propiciados por la emergencia sanitaria específicamente en los últimos dos años.

El grupo de participantes durante el pilotaje de la encuesta fue de 16 . De ellos, $75 \%$ son mujeres y $25 \%$ hombres. En cuanto al área de investigación en el que se desempeñan profesionalmente: $56.3 \%$ son del área de Ciencias Sociales, mientras que el restante $43.7 \%$ corresponde a otras áreas. Asimismo, del total, $62.5 \%$ están vinculados al área educativa.

\section{A. Financiamiento}

Los objetivos fundamentales del CONACYT son: 1) apoyar financieramente a los Centros Públicos de Investigación; 2) apoyar financieramente mediante un sistema de becas que impulse la formación de nuevos investigadores; 3) apoyar proyectos de investigación que consideren la problemática nacional, y 4) apoyar financieramente mediante un sistema de becas para investigadores de alto desempeño (Rivas, 2004). En este caso, el $100 \%$ de los participantes fueron o son becarios CONACYT y aproximadamente el $20 \%$ de ellos, también fueron becarios de otra institución.

En México los estudios de maestría y doctorado avalados por el CONACYT son de 24 y 48 meses de duración. Del total de los encuestados, el $88.23 \%$ requirió más tiempo del establecido (2 años) para finalizar y obtener su grado de maestría; mientras que el $33.33 \%$ requirió más tiempo para finalizar y obtener su grado de doctorado (3 años).

\section{B. Movilidad}

Cincuenta por ciento de los participantes han estudiado al menos un semestre en el extranjero y en una tercera parte de los casos se trata de estudios de posgrado. Del total, sólo un $12.5 \%$ tuvieron más de un periodo de estudios en el extranjero, siendo éstos: maestría y doctorado.

\section{Actividades de investigación}

En los últimos cinco años, las actividades de investigación consumieron un promedio de tres años; mientras que las actividades, dedicadas al trabajo de campo, académicas y de investigación, cerca de 3 años y medio. Esto podría implicar que la carga laboral es mayor para las actividades académicas que de investigación, y que, por otra parte, la carga laboral rebasaría las 8 horas de trabajo diario.

\section{Productividad}

Andrade-Molina, Montecino y Sánchez, (2020) mencionan que la calidad y la cantidad de las publicaciones se toman como indicador de la productividad de los académicos. La encuesta arrojó un promedio de publicaciones en revistas indexadas en el lapso de los últimos cinco años de 2.3 artículos (menos de uno por año). Sólo el $35.29 \%$ de los encuestados logran publicar un artículo de investigación en revistas indexadas, al año. Y debido a la perturbación causada por la pandemia de Covid-19, la revisión por pares de las revistas ha sufrido dificultades con los plazos asociados.

Por su parte, el promedio de participación en congresos nacionales es de aproximadamente cuatro congresos en el mismo periodo, mientras que en el caso de los congresos internacionales es de menos de 2 congresos.

\section{E. Gráfico de Trayectorias}

El análisis propone una manera de interpretar las trayectorias a través de la construcción de una herramienta gráfica en donde sea posible visualizarlas. Esta propuesta se encuentra aún en desarrollo, pero la interpretación de resultados de acuerdo a esta herramienta gráfica, estaría basada en un análisis temporal.

\section{F. Análisis cualitativo: Afectaciones por COVID-19}

Las afectaciones u obstáculos que se reportaron en la encuesta en los últimos cinco años, se presentan en la Tabla 1 en orden del mayor al menor número de menciones (porcentaje del total).

Tabla 1

Obstáculos enfrentados en los últimos cinco años

\begin{tabular}{clr}
\hline \multicolumn{1}{c}{ Aspecto } & Obstáculo & Menciones \\
\hline \hline \multirow{5}{*}{ Económico } & $\begin{array}{l}\text { Desempleo en el área } \\
\text { de investigación }\end{array}$ & $50 \%$ \\
\cline { 2 - 3 } Personal & Bajos sueldos & $31.2 \%$ \\
\cline { 2 - 3 } & Falta de financiamiento & $19 \%$ \\
\hline & Maternidad y crianza & $19 \%$ \\
\cline { 2 - 3 } Institucional & $\begin{array}{l}\text { Plabbing } \\
\text { organización y }\end{array}$ & $31.2 \%$ \\
\cline { 2 - 3 } & $\begin{array}{l}\text { Falta de atención a } \\
\text { necesidades educativas }\end{array}$ & $12.5 \%$ \\
\cline { 2 - 3 } & $\begin{array}{l}\text { Relación entre el } \\
\text { trabajo académico, de }\end{array}$ \\
& $\begin{array}{l}\text { investigación y } \\
\text { administrativo }\end{array}$ \\
\cline { 2 - 3 } & Seguridad & $6.25 \%$ \\
\hline
\end{tabular}

En ese sentido, el primer paso consistió en codificar las respuestas de los participantes, de acuerdo a criterios establecidos por dos investigadores de forma separada. Las afectaciones por emergencia sanitaria de COVID-19 que se mencionaron en la encuesta, se presentan en orden del mayor al menor número de menciones en la Tabla 2.

Tabla 2

Obstáculos por emergencia sanitaria por COVID-19

\begin{tabular}{|c|c|c|}
\hline $\begin{array}{r}\text { Aspecto } \\
\text { Institucional } \\
\end{array}$ & $\begin{array}{l}\text { Obstáculo } \\
\text { Procesos } \\
\text { administrativos } \\
\end{array}$ & Menciones \\
\hline \multirow{5}{*}{ Económico } & Disminución del salario & $31.2 \%$ \\
\hline & Gastos imprevistos & $12.5 \%$ \\
\hline & $\begin{array}{l}\text { Reducción del } \\
\text { financiamiento }\end{array}$ & $19 \%$ \\
\hline & $\begin{array}{l}\text { Actividades de } \\
\text { investigación }\end{array}$ & $25 \%$ \\
\hline & Estrés & $19 \%$ \\
\hline \multirow[t]{2}{*}{ Personal } & Mejoras & $19 \%$ \\
\hline & Desgaste físico & $6.2 \%$ \\
\hline No Aplica & Ninguno & $25 \%$ \\
\hline
\end{tabular}

Los dos principales obstáculos que enfrentan las investigadoras, están directamente vinculados con la cuestión económica. El primero de ellos es la falta de oportunidades laborales en el área de investigación, tanto en el ámbito privado 
como en el público. En este caso, la mitad de los participantes hicieron mención a esta situación. Las participantes aseguraron que la falta de oportunidades laborales afectaba directa y negativamente la movilidad profesional y en consecuencia de manera agravante el desempleo.

El segundo obstáculo, mencionado por una tercera parte de los participantes, fue los bajos sueldos; y en términos también económicos, se hizo mención del escaso financiamiento para actividades de investigación. A todo esto, la emergencia sanitaria por COVID-19 ha propiciado la reducción del ya insuficiente financiamiento en los ámbitos laboral y educativo, ya sea en forma de disminución de: becas, convocatorias, apoyo institucional para congresos $\mathrm{y} / \mathrm{o}$ viáticos. Asimismo, esta disminución en el financiamiento, incrementa la problemática original de desempleo en el área de la investigación. A esta coyuntura, dos situaciones económicas se agregan en los últimos dos años: los gastos imprevistos (médicos y tecnológicos), y la disminución de sueldo evidenciada en la reducción de jornadas y las horas de trabajo extra no remunerado para la adaptación a nuevas tecnologías de comunicación.

En un segundo nivel, se encuentran aquellos obstáculos que se presentan en el ámbito institucional, y que afectaron el desempeño profesional de los participantes en los últimos cinco años. En este rubro, se encuentran aspectos como el hostigamiento y acoso laboral (por parte de colegas y jefes), también denominado mobbing, los problemas de planeación y organización institucionales: que también se vieron drásticamente agravados por la emergencia sanitaria generando cambios fundamentales en los proyectos sin previo aviso e incluso una de ellas mencionó las implicaciones del cambio en el campo teórico que implica replantear los proyectos de investigación. $\mathrm{Y}$ por último la relación entre el trabajo académico, de investigación y el administrativo. En este sentido, a partir del 2020, con la emergencia sanitaria, la reducción de financiamiento repercutió negativamente al no facultar a las instituciones para generar convenios con otras organizaciones. Sin embargo, la mayor afectación se sufrió a nivel administrativo, por ejemplo, generando demoras en procesos de dictamen de artículos para publicación o trámites de titulación. También se percibió una disminución de la actividad de investigación implicando en muchos casos retrasos o cancelaciones de trabajo de campo.

En el tercer nivel, pero no menos importante, se mencionaron obstáculos relacionados a los aspectos personales siendo la principal mención el tiempo destinado a la maternidad y la crianza (x3). En términos generales, sin embargo, también se mencionaron afectaciones en el ámbito de la salud emocional a partir de la emergencia sanitaria como son el estrés y el agotamiento.

Por último, hubo un espacio para quienes consideraron que la emergencia no generó ningún obstáculo adicional (una cuarta parte de los participantes), e incluso quienes consideraron que se vieron de alguna manera beneficiados por la circunstancia, aunque sólo se mencionó la disminución de horas de traslado al centro de trabajo.

\section{CONCLUSIONES}

El presente proyecto es fácilmente transferible a otros contextos, sin embargo, sí es necesario tomar medidas necesarias y conocer el funcionamiento de las diversas instituciones nacionales e internacionales que proporcionan financiamiento al sector de la investigación en el área educativa.

Los resultados de este trabajo realizado en su fase piloto, serán la base para las adecuaciones a la encuesta y su próxima aplicación a nivel nacional. Por ejemplo, deben agregarse referencias socio-económicas de las participantes y condiciones específicas de etapas de desempleo durante su trayectoria profesional. Por otro lado, es posible profundizar en los aspectos positivos que la emergencia sanitaria pudo generar en los procesos de enseñanza-aprendizaje en los que estuvieron inmersas. Por último, un análisis comparativo y detallado desde la perspectiva de género permitiría definir las diferentes condiciones de los investigadores, y las propuestas pertinentes de acuerdo a su género.

\section{AgRAdECIMIENTOS}

Los autores y demás investigadores involucrados en esta investigación agradecen al Instituto Politécnico Nacional por el apoyo a esta investigación que lleva por nombre: "Análisis de trayectorias de estudiantes y egresados de nivel posgrado en México: retos y oportunidades en el marco de la emergencia sanitaria por COVID-19”, con clave del proyecto: $20211057 \mathrm{y}$, además agradecemos el apoyo de Hipatia México en la concreción de este proyecto.

\section{REFERENCIAS}

Andrea-Molina, M., Montecino, A., y Sánchez, M. (2020). Beyond quality metrics: defying journal rankings as the philosopher's stone of mathematics education research. Educational Studies in Mathematics. 103(3). 313-337.

Bonilla, M. (Ed) (2015). Diagnóstico del Posgrado en México: NACIONAL. Consejo Mexicano de Estudios de Posgrado, A. C. México. Recuperado de https://www.posgrado.unam.mx/sitios_interes/document os/comepo_regiones.pdf

CEPAL (Comisión Económica para América Latina y el Caribe). (2020a). "América Latina y el Caribe ante la pandemia del COVID-19: efectos económicos y sociales", Informe Especial COVID-19, No 1, Santiago, abril.

CEPAL. (2020b). "Pactos políticos y sociales para la igualdad y el desarrollo sostenible en América Latina y el Caribe en la recuperación pos-COVID-19”, Informe Especial COVID-19, No 8, Santiago, octubre.

Creswell, J. W., y Creswell J. D. (2018). Research design: qualitative, quantitative, and mixed methods approach. SAGE Publications, Inc.

Rivas, L. (2004). La formación de investigadores en México. Perfiles Latinoamericanos, 12(25), 89-113.

Sanz, I., Sáinz González, J., Capilla, A. (2020). Efectos de la Crisis del Coronavirus en la Educación. Organización de Estados Iberoamericanos para la Educación, la Ciencia y la Cultura (OEI). Recuperado en https://oei.org.br/arquivos/informe-covid-19d.pdf

The Chronicle of Higher Education. (2020). Moving Online Now. How to Keep Teaching during Coronavirus. Recuperado en https://connect.chronicle.com/rs/931EKA-218/images/CoronaVirus_ArticlesCollection.pdf 
Yi, Y., Lagniton, P., Ye, S., Li, E., y Xu, R. (2020). COVID-

19: what has been learned and to be learned about the novel coronavirus disease. International Journal of Biological Sciences. 16(10). 1753-1766. 